Randomised clinical trials guard against overoptimism likely to arise from uncontrolled experiments, where bias is easily introduced. It is not true that no major advances have arisen from randomised trials in obstetrics. Two important examples are the use of corticosteroids to prevent the respiratory distress syndrome and the use of anti-D to prevent rhesus isoimmunisation. Furthermore, several disasters have occurred because randomised trials were not' carried out, including the well known epidemic of retrolental fibroplasia. ${ }^{2}$ More generally, the widespread use of inferior research designs leads to confusion in published medical reports. Of course, it is known that a few people cheat when carrying out randomised trials, but that is a reason for trying to design trials in such a way as to make such fiddling impossible, not for abandoning clinical trials completely.

Randomised clinical trials are just one way of doing research and constitute a small minority of all research carried out. No one would say that all research should be in the form of randomised trials, but not to perform one when it is both possible and desirable is foolish and arguably unethical.

Clinical Research Centre,

D G AltMan Harrow, Middx HA1 3UJ

1 Sackett DL. Bias in analytic research. $\mathcal{F}$ Chronic Dis 1979;32: $51-63$.

2 Silverman WA. Human experimentation. A guided step into the unknown. Oxford: University Press, 1985.

SIR,-Professor D F Hawkins is right in saying that the randomised clinical trial is not appropriate for all clinical circumstances in obstetrics (23 January, p 291). Indeed, the whole point of our article (21 November, p 1298) was to show that this method was of limited use because of considerations of sample size and ethics. Professor Hawkins is also right to suggest that when the results of a randomised trial are not available or when randomisation is undesirable we should rely on alternative methods such as multivariate analysis (and logistic regression). These methods, however, are not equivalent to randomisation; they can adjust only for known variables and cannot remove unknown sources of bias. The latter often have a greater influence than the former on clinical outcome. ${ }^{1}$

Professor Hawkins is also correct to link the size of a proposed trial to the size of an effect that would influence clinical practice. An important point in our article, however, was the demonstration that the size of this difference is often very small indeed. We have shown this by the use of consumer questionnaires (involving the probability equivalence multiple gamble method), but this point must be obvious to almost all obstetricians, since the whole of our subject is now concerned with marginal increases in safety. For example, $50 \%$ of women would accept a doubling of the risk of miscarriage, from 1 in 200 to 1 in 100, to take advantage of early prenatal diagnosis by chorion biopsy. ${ }^{2}$ Thus to produce results with confidence limits which would be useful to only half of our prospective patients a randomised trial would need to be large enough to show a difference of this size.

Professor Hawkins is right to say that randomisation can pose ethical dilemmas. Again he is wrong if he seeks to imply that this inevitably precludes the use of proper, rigorous clinical experiments. Equally wrong are those who say that there are no ethical concerns and insist on randomisation until doubt is eliminated. We are seldom completely certain or completely uncertain, and for this reason the concept of equipoise was introduced. The less the degree of equipoise-that is, the more certain clinicians are about the advantages of one treatment over another-the less ethical does a trial become. If we stop a trial when $p$ is less than 0.05 then we regard it as unethical to randomise patients when there is $95 \%$ probability that one treatment is better. Most randomised trials involve a trade off between optimising outcome for an individual (by following the line of treatment which is thought to have the greates chance of success) and optimising outcome for society (thus providing the best possible unbiased data for future patients). It is inevitable, healthy, and appropriate that people will form differen opinions on this trade off.

Detailed analysis of the ethics of randomisation, bearing in mind the sample size required, the outcomes involved, and the perceived strength of existing evidence, is preferable to the use of slogans and entrenched positions on this issue.

RICHARD J LILFORD

Department of Obstetrics and Gynaecology,

St James's University Hospital,

Leeds LS9 7TF

1 Coronary Drug Project Research Group. Influence of adherence to treatment and response on cholesterol on mortality in the to treatment and response on cholesterol on mortality
coronary drug project. $N$ Engl f Med 1980;303:1038-41. 2 Thornton JG, Lilford RJ, Howel D. The safety of amniocentesis. Lancet 1986;ii:266.

\section{Medical confidentiality in child sexual abuse}

SIR,-A number of important issues were raised in Dr D T D Bulugahapitiya's letter (9 January, $p$ 133) on the paper by Dr Roger Williams, myself, and other colleagues on child sexual abuse (21 November, $p$ 1315).

Although the writer talks about Asian families, our experience is that these issues occur in every family we see. Many young people around the age of 14 manage to speak to a teacher they trust but ask that confidentiality should not be broken. This means that the professional confided in has a responsibility for that young person's welfare, and in my view the teacher quite rightly informed the doctor when the girl was admitted to hospital. The complaints were of abdominal pain - which can be associated with continuing traumatic experiences -and the teacher must have been relieved to share confidentiality. There should then perhaps have been a preliminary meeting as advised by the DHSS draft guidelines on interprofessional management so that those concerned could discuss the matter rapidly and decide how to proceed.

The psychiatrist's view that there should be no discussion of the abuse because of a risk of suicide was unhelpful because the opportunity to talk to the young person in detail was lost. It is entirely appropriate for a professional to say to a 14 year old that because of the magnitude of the problem he or she cannot maintain confidentiality and that the information must be shared with people who can ensure that she can be protected. It is a measure of the girl's desperation, perhaps her wish to draw further attention to the abuse, that she took an overdose of the very antidepressants that she had been prescribed. There is no information about a detailed interview with the girl to ascertain the extent of the abusive experiences or their effect on her. The fact that there are other young people living with the alleged abuser is of great concern. It is possible to examine even extremely shy young people if sufficient time is taken to sort out the young person's anxieties about inappropriate early sexual experiences.

Although the parents insisted on confidentiality and that the girl should not be seen by any other doctor except the psychiatrist and general practitioner, there is a question whether it is possible to grant such confidentiality, given the fact that other children in the community might be at risk. If medical staff take on the task of accepting strict confidentiality in these cases this may run counter to agreements about the management of abuse between professionals in the community, and this means that they are now protective agents to this child. This is a heavy responsibility.

Of course, there are concerns about professionals carrying out their tasks in an insensitive and uncaring way, but it is essential that professionals should educate each other and devise proper management strategies. Abuse occurs in every community and it is our responsibility to ensure that children are protected and that we learn to understand each community in enough detail to be able to provide proper protection and proper treatment.

This is another case where there could have been a tragedy if the overdose had been successful. The management of this case resembles the management that was prevalent for many years before people began to recognise abuse and its long term effects. It is sad when avoidance and secrecy are practised rather than openness and a willingness to learn how to manage these extremely difficult and worrying cases.

Department of Psychological Medicine,

ARnon Bentovim

Hospitals for Sick Children,

London WCIN 3JH

\section{Should colonoscopy be the first} investigation for colonic disease?

SIR,-While one would not necessarily argue with the primary conclusion of the paper by Drs D C Lindsay and others that radiological examination of the colon is not a necessary prerequisite for colonoscopy, it is unfortunate that those who read the article will come to the conclusion that radiology is seriously inferior to colonoscopy, even though the authors declare that this is not the intention.

The methods in the paper leave serious doubts about the validity of their comparison of colonoscopy and radiology. For example, colonoscopy was performed by four experienced and enthusiastic endoscopists, whereas radiology was performed by any one of a number of radiologists. Agreed, this is normal clinical practice, but we are not told whether the radiologists were informed that their investigations were being scrutinised for a prospective study or whether the protocol was discussed with the radiologists at the outset. The colonoscopists would undoubtedly have been aware that their patients were being prospectively assessed, and we can assume therefore that they made particular attempts to examine the colon as fully as possible and to give an adequate report. Unless the radiologist was also being given the same advantage the comparison becomes open to the influence of bias. Secondly, who scored the radiographs in an attempt to assess adequacy of examination? It takes some time to become experienced in interpreting the nuances of the barium enema examination, and scoring by a nonradiologist is quite unacceptable particularly if the same non-radiologist scores the colonoscopy examinations. Scoring by a clinician who is also a colonoscopist is open to considerable risk of bias, even though the clinician may make a particular attempt to avoid this. If the protocol was discussed with radiologists before the study, and if a radiologist did take part in the assessment of films, why is there no radiologist among the authors?

If a group of radiologists from the Royal Victoria Infirmary in Newcastle were to examine the films and reports of the barium enemas and the colono- 
scopy reports of the 168 patients included in Lindsay's study, would they come to the same conclusions and would the average colonoscopist believe it?

Gastroenterology Unit, University Hospital of South Manchester, Manchester M20 8LR

SIR,-In the study by Dr D C Lindsay and colleagues the fact that only $36 \%$ of barium enema examinations were considered to be complete is disturbing. Could this be related to the preparation technique, in which the barium examination was done immediately after the cleansing enema although this goes against the recommendation that at least one hour should elapse to allow residual water to be resorbed by the colon, thus allowing optimal mucosal coating?' Perhaps it would have been helpful to have had the direct cooperation of a radiologist in this study rather than physicians alone assessing the adequacy of the barium enema by reviewing the films and the radiologist's report.

The authors stated that patients aged under 40 in whom the expectation of disease was low were excluded from the study, yet patients as young as 19 and 20 years of age were included in both the study groups. It would be interesting to know whether subjective or objective methods were useds to predict the presence or absence of disease.

There were 29 patients in whom the barium enema examination was considered incomplete and yet in whom the "exclusion of gross disease was thought to preclude the need for further large bowel investigation." It would be interesting to know whether polyps were considered to be gross disease or to be excluded by an incomplete examination.

Department of Radiology

St Thomas's Hospital,

London SE1 7EH

1 Kendrick RGM, Mackenzie S, Beckley DE. A comparison of four methods of bowel preparation for barium enema. Clin Radiol 1981:32:95-7.

SIR,-The article by Dr D C Lindsay and others (16 January, $p$ 167) on primary investigation of the large bowel by barium enema or colonoscopy raises a number of relevant issues.

Firstly, the methods implies that bowel preparation was identical for both groups. This is not so as patients at both the Royal Victoria Infirmary and Freeman Hospital in Newcastle do not receive a cleansing enema before a barium enema. Dietary preparation and sodium picosulphate (Picolax) only are used.

Secondly, colonoscopies were performed by "experienced colonoscopists" (authors' words) and barium enemas mostly by consultant radiologists. We would be interested to know what is meant by the term "experienced" since only one of the authors is a consultant.

Thirdly, the grading of the quality of the examinations is not specified and there is no evidence to suggest that any interobserver analysis of assessments was undertaken.

Fourthly, from the authors' data adequate visualisation of the bowel was achieved in $83 \%$ of enemas but in only $81 \%$ of colonoscopies. Yet they claim that "complete" examinations were seen in only $36 \%$ of the enemas and in $67 \%$ of the colonoscopies. Perhaps they could comment on the apparent inconsistencies.

Fifthly, the data on the necessity for a second diagnostic procedure do not appear to follow predetermined criteria. Was a detailed protocol of indications for a second diagnostic procedure drawn up?

Finally, two impassable strictures were found in the 20 incomplete colonoscopies with no subsequent barium enema. Surgeons usually like the assurance that only a single lesion is present before embarking on a major procedure. Barium will pass through a very small orifice and these two cases were inadequately investigated.

We are saddened that clinical colleagues should choose to undertake and have published a clinical study employing our professional services without consultation or acknowledgment.

\section{P M HACKING} J P OWEN

Department of Radiology,

Royal Victoria Infirmary,

Newcastle upon Tyne NE1 4LP

A BURRIDGE

AUTHORS' REPLY,-Many of the points made by your correspondents are perfectly valid, particularly about the enthusiasm of the colonoscopists conducting the study compared with the radiologists, most of whom were unaware of the pro spective comparison.

The findings of the two investigations were collated from the reports filed in the patients' case records, but as clinicians we attempted to evaluate retrospectively the completeness and thus diag nostic certainty of the examinations. Your corres pondents and several colleagues have commented that this non-radiological evaluation was less than ideal, and indeed the results of the completeness of the examinations might have been quite differen had the comparison been carried out by radiologists. In defence we wish to emphasise that the study was designed to be an evaluation of clinical practice as carried out in our hospitals, and we do not believe that our overall conclusion that colonoscopy is an acceptable first line investigation of the colon requires modification.

As Dr Hacking and colleagues point out, cleans ing enemas are no longer carried out before barium enema, and we regret the error in our methods section, which should have read: "The barium studies were carried out by several different radiologists, but most were not consultants."

\section{COBDEN \\ C O RECORD}

Royal Victoria Infirmary

Newcastle upon Tyne NE1 4LP

SIR,-Dr D C Lindsay and others (16 January p 167) proposed that total colonoscopy should be the initial investigation of choice for patients with large bowel symptoms. We consider it unfortunate that rigid sigmoidoscopy rather than fibreoptic sigmoidoscopy was used in conjunction with double contrast barium enema examination. There was no mention of findings which arose from sigmoidoscopy, which were presumably of no or limited value. If fibreoptic sigmoidoscopy had been used instead 48 of the 56 patients would have been diagnosed correctly.

We have recently been evaluating our experience of 446 colonoscopies carried out over the past 21 months with particular emphasis on patients in the pensionable age group. It is our impression that colonoscopy, particularly in the older more frail patients, is poorly tolerated compared with barium enema examination and is not the ideal first line investigation for patients with large bowel disease.

Our preferred approach is to use a double contrast barium enema examination as the firs investigation, except in those patients who have had rectal bleeding, when we advise fibreoptic examination. The widely held belief that reaching the caecum is the gold standard in total colonoscopy has been exaggerated, particularly in patients who are elderly, who often have indifferent bowel preparation and in whom heavy sedation is hazardous. Although a barium enema examination may not show the right colon well, if a reasonably good quality film has been obtained of the right colon and caecum then few important lesions will be missed even in these patients.

If we adopted total colonoscopy as the first line investigation we would need five colonoscopy sessions a week to deal with the numbers referred. Clearly this is an impracticable proposition for the average district general hospital, particularly as few have more than one or two dedicated colonoscopists. Total colonoscopy, however, is mandatory in surveillance for polyps and in patients with longstanding inflammatory bowel disease.

We have been very impressed with the value of fibreoptic sigmoidoscopy, performing 250-300 examinations a year in our endoscopy unit and a further 150-200 examinations on outpatients. The procedure is well tolerated in all age groups, little bowel preparation is necessary, and a high diagnostic yield results. It is very cost effective, can be easily learnt by junior staff, and occasionally reduces the need for either a double contrast barium enema examination or total colonoscopy. Unfortunately, it is not available in many hospitals because of lack of finance. We believe that with modern fibreoptic instruments available there is little or no need for rigid sigmoidoscopy in assessing large bowel disease, in the same way that we no longer carry out rigid oesophagoscopy for patients who have dyspepsia.

\section{J SNAPE}

A C B WICKS

Leicester General Hospital

Leicester LE5 4PW

SIR,-I agree with Dr D C Lindsay and others (16 January, p 167) that, providing finance and manpower are available, colonoscopy will probably increasingly replace double contrast barium enema in the investigation of patients referred with suspected colonic or rectal disease. Can I emphasise, however, the importance of continuing to carry out rigid sigmoidoscopy on these patients when they are first seen at the outpatient clinic.

Firstly, when the colonoscope or flexible sigmoidoscope is inserted it is frequently $10-15 \mathrm{~cm}$ inside the rectum before any adequate view is obtained. When the fibreoptic scope is withdrawn it is often difficult to obtain adequate views of the whole of the rectal ampulla as the scope tends to slip out very quickly. The rectal ampulla is most adequately covered by the rigid sigmoidoscope.

Secondly, both flexible sigmoidoscopy and colonoscopy are usually preceded by cleansing of the bowel using oral preparations, rectal enemas, or both. This can impart to the rectal mucosa a degree of reddening and it is quite impossible to differentiate between this and a true mild proctitis. This problem does not arise with sigmoidoscopy carried out with a rigid instrument, for which the bowel may be unprepared.

Thirdly, if an enema or oral preparation is given to clean the rectum then a very valuable physical sign may be missed-that is, a small amount of dark red blood seen mixed in the bowel motion, which indicates the presence of definite disease higher in the bowel.

As most of these patients are seen at an outpatient surgical clinic dealing specifically with gastrointestinal disease the rigid instrument is readily available and the whole procedure adds perhaps only one to two minutes to the routine 